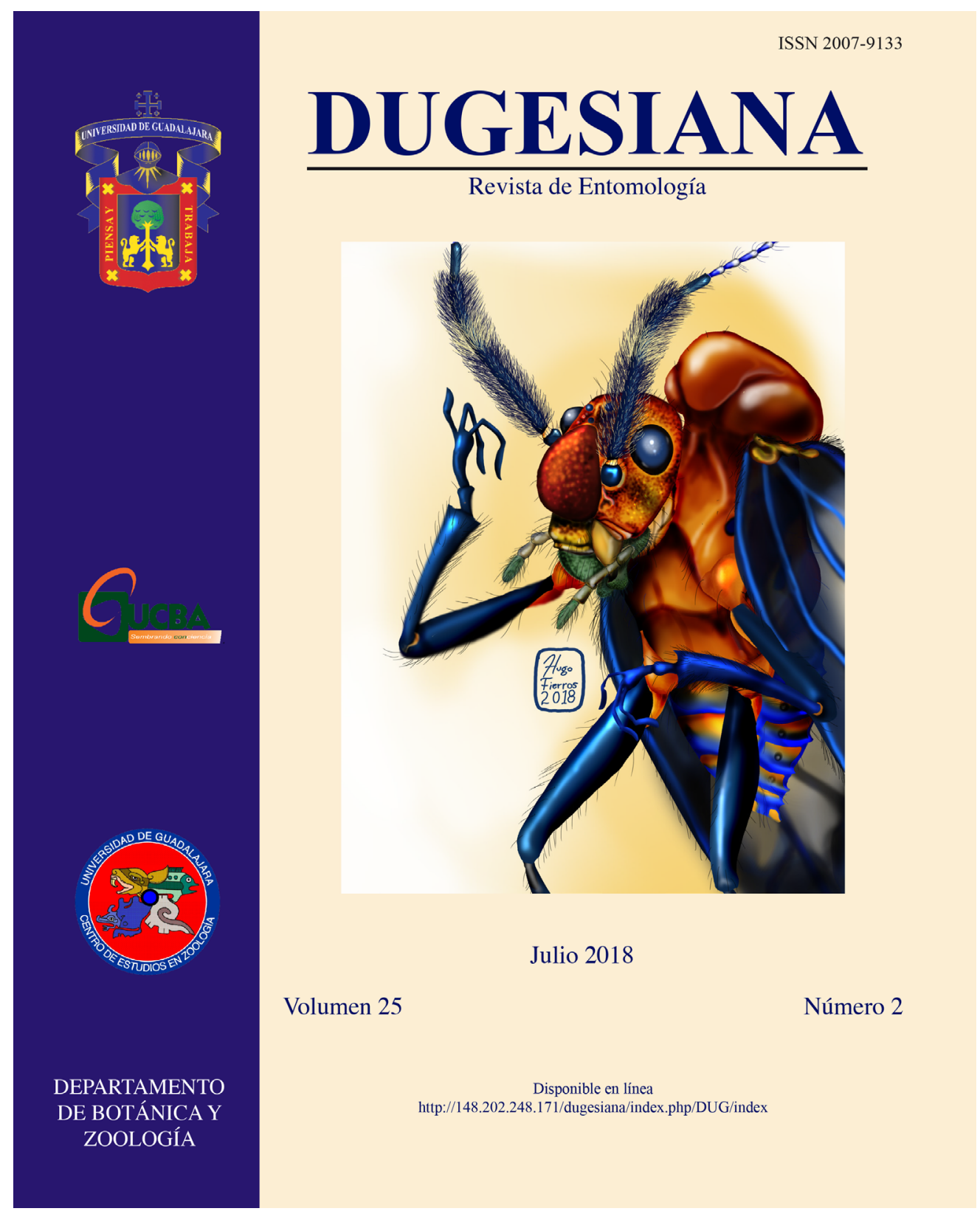

Dugesiana, Año 25, No. 2, julio 2018-diciembre 2018 (segundo semestre de 2018), es una publicación semestral, editada por la Universidad de Guadalajara, a través del Centro de Estudios en Zoología, por el Centro Universitario de Ciencias Biológicas y Agropecuarias. Camino Ramón Padilla Sánchez \# 2100, Nextipac, Zapopan, Jalisco, Tel. 37771150 ext. 33218, http://148.202.248.171/dugesiana/index.php/DUG/index, glenusmx@gmail.com. Editor responsable: José Luis Navarrete Heredia. Reserva de Derechos al Uso Exclusivo 04-2009-062310115100203, ISSN: 2007-9133, otorgados por el Instituto Nacional del Derecho de Autor. Responsable de la última actualización de este número: José Luis Navarrete Heredia, Editor y Ana Laura González-Hernández, Asistente Editorial. Fecha de la última modificación 25 de julio 2018, con un tiraje de un ejemplar.

Las opiniones expresadas por los autores no necesariamente reflejan la postura del editor de la publicación.

Queda estrictamente prohibida la reproducción total o parcial de los contenidos e imágenes de la publicación sin previa autorización de la Universidad de Guadalajara. 


\section{Descripción de Amphibolips cibriani Pujade-Villar n. sp. para México (Hymenoptera: Cynipidae: Cynipini)}

\section{Description of Amphibolips cibriani Pujade-Villar n. sp. from México (Hymenoptera: Cynipidae: Cynipini)}

\section{Juli Pujade-Villar ${ }^{1 *}$, Uriel M. Barrera-Ruíz ${ }^{2} \&$ Víctor Cuesta-Porta $^{1}$}

${ }^{1}$ Universitat de Barcelona, Facultat de Biologia, Departament de Biologia Evolutiva, Ecologia i Ciències Ambientals, Avda. Diagonal 645, 08028-Barcelona, Catalunya. ${ }^{2}$ Instituto de Fitosanidad, Colegio de Postgraduados, Km 36.5 Carretera México-Texcoco. 56230-Montecillo, Estado de México, México. E-mail: jpujade@ub.edu, umbr757@ gmail.com, victorcp93@gmail.com.*Autor de correspondencia

\section{RESUMEN}

Se describe de México una nueva especie de cinípido de encino, Amphibolips cibriani Pujade-Villar n. sp., conocida sólo a partir de su generación asexual que induce agallas en las yemas de Quercus crassipes Humb. \& Bonpl. (Sección Lobatae). Se dan datos referentes a la diagnosis, distribución y biología de esta nueva especie.

Palabras clave: Hymenoptera, Cynipidae, Cynipini, Amphibolips, nueva especie, México, Quercus crassipes.

\section{ABSTRACT}

A new species of oak gallwasps, Amphibolips cibriani Pujade-Villar n. sp. known from the asexual generation only is described from Mexico inducing galls on buds of Quercus crassipes Humb. \& Bonpl. (Section Lobatae). Diagnosis data, distribution and biology of the new species are given.

Key words: Hymenoptera, Cynipidae, Cynipini, Amphibolips, new species, Mexico, Quercus crassipes.

La tribu Cynipini (Hym., Cynipidae), caracterizada por producir agallas en Fagaceae, incluye un total 34 géneros con cerca de 1000 especies distribuidas en las regiones Holárctica, Neotropical y Oriental (Ronquist et al., 2015). El género Amphibolips Reinhard, 1865 se distribuye exclusivamente en el continente americano, desde Canadá hasta Panamá, por lo que está presente tanto en la región Neártica como Neotropical.

Amphibolips incluye un total de 52 especies: 30 de EEUU y Canadá, 18 de México, 1 de México y EEUU (Arizona) y 3 de Panamá (Burks 1979; Melika \& Abrahamson 2002; Medianero \& Nieves-Aldrey 2010; Pujade-Villar \& FerrerSuay, 2015). Todas las especies conocidas de Amphibolips están asociadas con especies de Quercus de la sección Lobatae (robles rojos).

Kinsey (1937) propuso complejo 'niger' para incluir seis de las ocho nuevas especies que describió. El complejo 'niger' está caracterizado por producir agallas redondeadas, de aspecto lanoso con una estructura central muy dura y leñosa que contiene la cámara larval y adultos caracterizados por ser formas agámicas con antenas de 16-17 segmentos, mesonoto algunas veces con carenas longitudinales y mesoscutelo redondeado posteriormente. Las especies mexicanas que pertenecen al complejo 'niger' son: A. elatus Kinsey, 1937; A. jubatus Kinsey, 1937; A. maturus Kinsey, 1937; A. nebris Kinsey, 1937; A. niger Beutenmüller, 1911 y A. pistrix Kinsey, 1937.
En contraposición a este complejo, Melika et al. (2011) propusieron el complejo 'nassa' para el resto de especies del género, caracterizadas por producir agallas esféricas, globosas o fusiformes, nunca pubescentes, con un parénquima esponjoso que rodea la cámara larval central o a veces ésta soportada por filamentos radiantes, con adultos tanto sexuados como presumiblemente agámicos, con 15 segmentos en la antena de los machos y 13-14 en las hembras, mesoescudo siempre con una escultura gruesa rugosa o rugoso-reticulada y mesoscutelo emarginado posteriormente. Trece especies mexicanas se incluyen en este complejo: A. dampfi Kinsey, 1937; A. durangensis Nieves-Aldrey \& Maldonado, 2012; A. fusus Kinsey, 1937; A. hidalgoensis Pujade-Villar \& Melika, 2011; A.jaliscensis Nieves-Aldrey \& Pascual, 2012; A. malinche NievesAldrey \& Pascual, 2012; A. michoacaensis Nieves-Aldrey \& Maldonado, 2012; A. nassa Kinsey, 1937; A. nevadensis Nieves-Aldrey \& Pascual, 2012; A. oaxacae Nieves-Aldrey \& Pascual, 2012; A. palmeri Bassett, 1890; A. tarasco Nieves-Aldrey \& Pascual, 2012 y A. zacatecaensis Melika \& Pujade-Villar, 2011.

En este trabajo se describe una nueva especie de Amphibolips del grupo 'nasa' que produce agallas relativamente duras, esféricas, con superficie rugosa y uniloculares colectadas en Q. crassipes Humb. \& Bonpl. (sección Lobatae). 


\section{MATERIAL Y MÉTODOS}

Las agallas fueron colectadas en el Fraccionamiento Bosques de Santa Fe, un desarrollo residencial localizado en la delegación Cuajimalpa, al poniente de la Ciudad de México sobre $Q$. crassipes Humb. \& Bonpl., roble que pertenece a la sección Lobatae (Govaerts \& Frodin, 1998). Fueron conservadas en el laboratorio en espera de la obtención de los adultos, siempre hembras agámicas.

Se siguió la terminología de las estructuras morfológicas de Liljeblad \& Ronquist (1998) y Melika (2006). Las abreviaturas de la venación del primer par de alas según Ronquist \& Nordlander (1989) y la terminología superficial cuticular según Harris (1979).

Las mediciones y abreviaturas usadas incluyen F1F10, 1er y siguientes flagelómeros o bien antenómeros para los últimos segmentos (A13 y A14); POL (distancia post-ocelar) la distancia entre los márgenes interiores de los ocelos posteriores; OOL (distancia ocular-ocelar) la distancia desde el borde exterior de un ocelo posterior hasta el margen interno del ojo compuesto; LOL, la distancia entre los ocelos laterales y frontales. La anchura de la celda radial se mide desde el margen del ala hasta la vena Rs.

Las imágenes SEM fueron realizadas por el primer autor, utilizando un microscopio electrónico de barrido ambiental (FEI Quanta 200 ESEM) sin cubrir de oro los especímenes.

El material tipo ha sido depositado en las siguientes instituciones: UB, Universidad de Barcelona, Cataluña (J. Pujade-Villar); UACh, Universidad Autónoma Chapingo, Estado de México, México (D. Cibrián-Tovar), AMNH, American Museum of Natural History, New York, USA (J. M. Carpenter); y USNM, U.S. National Museum of Natural History, Smithsonian Institution, Washington, DC, USA (M. Buffington).

\section{RESULTADOS Amphibolips cibriani Pujade-Villar n. sp. (Figs. 1-4) \\ http://zoobank.org/049B75E2-DEF1-4EF7- A106-D78258701712}

Material tipo. Holotipo $q$ depositado en la col. JP-V (UB) con las siguientes etiquetas: "MEX-316, Sta. Fe (Cuajimalpa, Ciudad de México), 19²1' 0.99" N -99¹6' 27.02" O" (etiqueta blanca), Q. crassipes, $2630 \mathrm{msnm}$., (25. iv.2017) 10-20.v.2017", col. DCT-2906" (etiqueta blanca); "Holotype Amphibolips cibriani Pujade-Villar n. sp., desig. JP-V 2018" (etiqueta roja). Paratipos (6̆): mismos datos que el holotipo (2ǒ, UB); (15.vi.2014) 15-20.v.2014: (2ǒ; 1ǒ AMNH y 1ǒ USNM); (23.iv.2017) 20-25.v.2017: (2ǒ, UACh).

Material adicional. Mismos datos holotipo, (30.v.2013) extrac. 31.v.2013: 3ǒ pupas; MEX-299, (20.vii.2014) extrac. 29.vi.2015: 2ǒ (fragmentos) + 2ǒ pupas.

Diagnosis. Según las descripciones y las claves de Nieves-Aldrey et al. (2012), Amphibolips cibriani PujadeVillar n. sp., atendiendo a la morfología alar, se aproxima
A. castroviejoi (de Panamá) y A. durangensis (de México) por presentar una banda clara transversal ancha que alcanza el margen inferior del ala. De A. castroviejoi se diferencia por la ausencia de notaulos y la presencia de arrugas en el propodeo. De $A$. durangensis por la escultura de la cabeza y del mesoscudo (menos homogénea y más gruesa en la nueva especie), por presentar las fosetas separadas por una carena central y por presentar las carenas del propodeo diferenciadas. De ambas especies se separa por la coloración del metasoma (rojizo en la nueva especie, negro o muy oscuro en $A$. castroviejoi y $A$. durangensis). Las agallas son esféricas y lisas en $A$. castroviejoi, fusiformes en $A$. durangensis y esféricas con superficie rugosa en la nueva especie.

Descripción. Hembra agámica.

Longitud. 5.3-5.4 $\mathrm{mm}(\mathrm{n}=4)$.

Color (Fig. 4d-e). Cabeza, mesosoma, antenas y patas negras; mandíbulas rojizas, dientes negros; clípeo castaño. Metasoma rojizo. Primer par de alas oscuras, con una banda transversal ancha que se extiende desde el tercio apical de la celda radial al margen inferior del ala; celda basal no infuscada.

Cabeza (Figs. 1a-b). En vista frontal 1.3 veces más ancha que alta, gena ligeramente ensanchada detrás del ojo. Cara inferior rugosa con los interespacios coriáceos, poco pubescente. Clípeo trapezoide, con una escultura coriácea, prominente en la parte superior; margen ventral sobresaliendo fuertemente sobre las mandíbulas y ligeramente sinuoso. Fosetas tentoriales anteriores inconspicuas; surco epistomal y líneas clípeo-pleurostomal levemente visibles. Estrías irradiantes de clípeo poco diferenciadas. Espacio malar 0.6 veces la altura del ojo compuesto. Distancia transfacial mayor a la altura del ojo (23:17). Diámetro del torulus (incluyendo el margen) mayor a la distancia entre los toruli (19:7); distancia entre el torulus y el borde interno del ojo menor que el diámetro del torulus (13:19). Los márgenes interiores de los ojos ligeramente convergentes. Gena rugoso-reticulada, interespacios coriáceos, pubescente, mayor que el diámetro del ojo en visión lateral. Vertex y frente con una escultura fuerte rugoso-reticulada, interespacios coriáceos, glabros. En la vista dorsal aproximadamente 2.5 veces más ancha que larga. POL tan largo como OOL; OCO muy estrecho menos de la mitad del diámetro del ocelo lateral; el ocelo lateral se separa de la órbita interna del ojo en 1.6 veces su diámetro más largo; relación POL: OOL: LOL igual a 8: 8: 2 , siendo el diámetro relativo del ocelo igual a 5 .

Antena (Fig. 1c). Antenas casi tan largas como la longitud de la cabeza + mesosoma (95:90), con 13 o 14 antenómeros; último segmento (A13+A14) variable, a veces parcialmente dividido en dos segmentos o casi completamente dividido. Pedicelo ligeramente más corto que ancho y la mitad de la longitud del escapo. El flagelo no se ensancha hacia el ápice, con setas cortas y aplicadas; sensílias placodeas densas y poco visibles, presentes de F4F11, ocupando toda la longitud del antenómero. F1 recto o 
muy ligeramente curvado, más estrecho basalmente, más largo que el escapo + pedicelo y más largo que F2 (1.3 veces). Fórmula antenal: 18: 11(x13): 37: 28: 23: 20: 18: 17: 16: 15: 15: 14: $30(15+15)$.

Mesosoma (Figs. 2a-c). En vista lateral un poco más largo que alto. Pronoto, moderadamente pubescente con una escultura rugoso-reticular irregular, carenado en la mitad inferior. Pronoto medialmente corto; relación de la longitud del pronoto medialmente / lateralmente $=$ 1/7. Placa pronotal indistinta dorsalmente. Mesoescudo escasamente pubescente y con una escultura rugosoreticulada irregular con interespacios coriáceos. Notaulos indiferenciados entre la escultura, a veces parcialmente visibles. Impresión mediana superficial poco diferenciada entre la escultura, pero visible desde la parte anterior del mesoscutum hasta la parte posterior, cruzada por arrugas transversales. Líneas paralelas anteriores visibles, extendiéndose hasta cerca de la mitad del mesoescudo; líneas parapsidales presentes extendiéndose hasta alcanzar el nivel anterior de la tégula; carena parascutal visible alcanzando el nivel del margen anterior de la tégula. Fisura transescutal estrecha. Mesoescutelo subcuadrado, aproximadamente 0.6 tan largo como mesoscutum. Fosetas escutelares subcuadradas ocupando $1 / 3$ parte la longitud del mesoscutelo, separadas medialmente por una carena, profundas, cruzadas por carenas irregulares transversales, intervalos lisos y márgenes posteriores indistintos. Mesoscutellum fuertemente reticulado-rugoso, moderadamente emarginado en el margen posterior; la emarginación posterior alcanza aproximadamente $1 / 5$ de la longitud del escutelo; en visión lateral sobrepasa un poco el metaescutelo. Axílula moderadamente pubescente, con márgenes anterior y posterior marcados. Mesopleuron moderadamente pubescente, rugoso-carenado. Surco metapleural poco evidente entre la escultura, pero visible, alcanzando aproximadamente la mitad de la altura de la mesopleura. Metascutellum coriáceo-rugoso; fosetas metanotales alutáceas y pubescentes; carena metanotal estrecha (1/5 parte de la altura de la foseta), coriácea. Propodeo reticulado-rugoso con interespacios coriáceos, densamente pubescente en los laterales; carenas laterales poco diferenciadas entre la escultura del propodeo, pero visibles, delimitando un área glabra, con tendencia a ser curvadas, Nucha lisa dorsalmente, carenada en los laterales.

Patas. Densamente pubescente; fémures y tibias robustos; metafemur 4.3 veces tan largo como ancho, curvado ventralmente e hinchado dorsalmente. Metatibia unas 5 veces más larga que ancha y más larga que el fémur (65:57). Metatarso más largo que los 3 tarsos siguientes y más corto que el resto de tarsómeros juntos; margen apical de los metatarsómeros 1-4 con setas erectas largas y fuertes. Uñas tarsales con un diente basal triangular fuerte (Fig. 2d).

Alas (Fig. 4b). Pubescentes en la superficie y en el margen. Más largas que la longitud el cuerpo (54:47). Venas R1 y Rs lejos del margen alar, muy poco pigmentadas; celda radial completamente abierta, alrededor 3.5 veces más larga que ancha (al proyectar la vena Rs); areola de tamaño moderado, triangular. Rs $+\mathrm{M}$ alcanzando la vena basal en su altura media. Primera abscisa de radio (2r) débilmente angulada, sin proceso medial interno claro.

Metasoma (Fig. 3). Casi tan largo como la cabeza y el mesosoma juntos (37:40), en vista lateral, tan alta como ancha. Segundo tergito metasomal cubriendo aproximadamente dos tercios del metasoma, con banda de micropuntuación claramente visible en un tercio posterior; siguientes tergitos micropuntuados; área ventral del segundo tergito metasomal moderadamente pubescente, setas escasas y dispersas en el tercio central latero-superior. Proyección de la espina del hipopígio larga, aproximadamente 7 veces más larga que ancha, con setas más largas que el ancho de la espina, pero sin formar un penacho apical (Fig. 3b).

Agallas (Figs. 4a-b). Uniloculares resultantes de la deformación de una yema. Esféricas (2-4 $\mathrm{cm}$ de diámetro), a menudo acabadas en punta y débilmente pedunculadas. La superficie es rugosa destacando un gran número de protuberancias. Al principio de color verde para ser marrón claro en la madurez. De consistencia dura. En un corte transversal se observa la cámara larval central lignificada (3 $\mathrm{mm}$ de diámetro $+1 \mathrm{~mm}$ de grosor la pared de la agalla interna) rodeada de un tejido esponjoso denso.

Huésped. Quercus crassipes Humb. \& Bonpl. (sección Lobatae). Especie endémica de México, presente en múltiples Estados (Valencia-A, 2004).

Distribución. México (Ciudad de México).

Biología. Especie conocida sólo por la generación asexual. Los adultos emergen a mediados de mayo. Estas agallas se encuentran altamente atacadas por inquilinos que se disponen en la cámara del inductor la cual aumenta mucho de tamaño y se compartimenta (Fig. 4c). La especie detectada es Synergus striatifrons Pujade-Villar and Lobato-Vila; el huésped de los Synergus obtenidos de Amphibolips sp (MEX-299) mencionados en Lobato-Vila \& Pujade-Villar (2017), corresponde a A. cibriani n. sp.

Etimología. Especie dedicada a nuestro amigo David Cibrián Tovar, colector de la agalla.

\section{DISCUSIÓN}

Los Amphibolips del grupo 'nassa' fueron divididos por Nieves-Aldrey et al. (2012) en tres grupos morfológicos atendiendo al aspecto del primer par de alas: (i) alas hialinas con una mancha oscura en el área basal de la celda radial, (ii) alas ahumadas con una banda clara desde la parte apical de la celda más o menos extendida hacia el margen inferior del ala, y (iii) alas oscurecidas sin una banda clara, a veces con una mancha incolora clara en la parte apical de la celda radial que no se extiende por debajo ésta. El primer grupo solo incluye una especie de Panamá, A. aliciae. El tercer grupo incluye la mayor parte de especies, algunas de ellas necesitan ser revisadas ya que en el trabajo mencionado se abusa de la coloración de las alas para diferenciar especies. El segundo grupo incluye además de la especie que aquí se describe, A. fuscus (con la banda transversal sin llegar al 
margen alar) y, A. damphi, A. durangensis y A. castroviejoi (con la banda clara mucho más larga). Las diferencias entre las dos últimas especies y A. cibriani n. sp. están expuestas en la diagnosis. Amphibolips dampfi presenta un escutelo marcadamente emarginado posteriormente (forma de V en visión dorsal), con una proyección en forma de cuerno afilado en visión lateral y la banda alar clara no llega al margen inferior.

La nueva especie se separa de todas las especies del género por el modelo de agalla. No existe ninguna especie con la superficie verrugosa. Por otro lado, las agallas son relativamente duras, esféricas y no se deforma con la presión, siendo, el resto de especies de agallas esféricas, más o menos fácilmente deformables.

Por otro lado, Nieves-Aldrey et al. (2012) consideraron que el grupo 'nassa' correspondía a formas sexuales mientras que el grupo 'niger' eran formas agámicas. Nosotros dudamos de que el grupo 'nassa' sea exclusivo de formas sexuales ya que después de colectar diversos años $A$. hidalgoensis en distintos estados mexicanos hemos obtenido centenares de ejemplares y nunca machos. En Santa $\mathrm{Fe}$, y en el mismo $Q$. crassipes del que describimos la nueva especie, hemos colectado unas agallas frágiles, delgadas y largas de las que hemos obtenido un macho morfológicamente cercano a la especie que aquí se describe. Por ello, consideramos que $A$. cibriani n. sp. se trate de una forma agámica y que posiblemente la agalla alargada mencionada pueda ser su forma sexuada. El aspecto de estas agallas sexuales recuerda a las que produce Tetramesa stipae De Stefani, 1901 (Hym. Chalcidoidea: Eurytomidae) en Stipa (Poaceae).

\section{AGRADECIMIENTO}

Este trabajo ha sido realizado en reconocimiento del entomólogo mexicano David Cibrián Tovar (Universidad Autónoma Chapingo) por su trayectoria y por tener la fortuna de haber colaborado con él en múltiples estudios de Cynipidae mexicanos. Estamos en deuda con la Dra. Silvia Romero Rangel (Universidad Nacional Autónoma de México) por la paciencia y comentarios referente a las especies de Quercus mexicanos.

\section{LITERATURA CITADA}

Burks, B.D. 1979. Superfamily Cynipoidea. In: Krombein, K.V., Hurd, P.D., Jr., Smith, D.R. \& Burks, B.D. (Eds.), Catalog of Hymenoptera in America of North of Mexico. Volume 1. Symphyta and Apocrita. Smithsonian Institution Press, Washington, DC, pp. 1045-1107.

Govaerts, R. \& Frodin, D.G. 1998. World Checklist and Bibliography of Fagales. Kew: Royal Botanic Gardens, Kew.
Harris, R., 1979. A glossary of surface sculpturing. State of California, Department of Food and Agriculture, Occasional Papers in Entomology, 28: 1-31.

Kinsey, A.C. 1937. New Mexican gall wasps (Hymenoptera, Cynipidae). II. Revista de Entomologia, 7 (4), 428-471.

Liljeblad, J. \& Ronquist, F. 1998. A phylogenetic analysis of higher-level gall wasp relationships (Hymenoptera: Cynipidae). Systematic Entomology, 23: 229-252.

Medianero, E. \& Nieves-Aldrey, J.L. 2010. The genus Amphibolips Reinhard (Hymenoptera: Cynipidae: Cynipini) in the Neotropics, with description of three new species from Panama. Zootaxa, 2360: 47-62.

Melika, G. 2006. Gall Wasps of Ukraine. Cynipidae. Vestnik zoologii, supplement, 21(1-2): 1-644.

Melika, G. \& Abrahamson, W.G. (2002) Review of the World Genera of Oak Cynipid Wasps (Hymenoptera: Cynipidae: Cynipini). Pp. 150-190. In: Melika, G. \& Thuróczy, Cs. (eds) Parasitic Wasps: Evolution, Systematics, Biodiversity and Biological Control. Agroinform, Budapest.

Melika, G. Equihua.Martínez, A. Estrada-Venegas, E.G., Cibrián-Tovar, D., Cibrián-Llanderal, V.D. \& PujadeVillar, J. 2011. New Amphibolips gallwasp species from Mexico (Hymenoptera: Cynipidae). Zootaxa, 3105: 4759.

Lobato-Vila, I. \& Pujade-Villar, J. 2017. Description of five new species of inquiline oak gall wasps of the genus Synergus Hartig (Hymenoptera, Cynipidae: Synergini) with partially smooth mesopleurae from Mexico. Zoological Studies, 56: 36, 28 pp. Doi:10.6620/ ZS.2017.56-36

Nieves-Aldrey, J. L., Pascual, E., Maldonado-López, Y., Medianero, E. \& Oyama , K. 2012. Revision of the Amphibolips species of Mexico excluding the "niger complex" Kinsey (Hymenoptera: Cynipidae), with description of seven new species. Zootaxa, 3545: 1-40.

Pujade-Villar, J. \& Ferrer-Suay, M. 2015. Adjudicació genèrica d'espècies mexicanes d'ubicació dubtosa descrites per Kinsey i comentaris sobre la fauna mexicana (Hymenoptera: Cynipidae: Cynipini). Butlletí de la Institució Catalana d'Història Natural, 79: 7-14.

Ronquist, F. \& Nordlander, G. 1989. Skeletal morphology of an archaic cynipoid, Ibalia rufipes (Hymenoptera: Ibaliidae). Entomologica Scandinavica, supplement 33, $1-60$.

Ronquist, F., Nieves-Aldrey, J. L., Buffington, M. L., Liu, Z., Liljeblad, J. \& Nylander, J. A. A. 2015. Phylogeny, Evolution and Classification of Gall Wasps: The Plot Thickens. PLoS ONE, 10(5): e0123301.

Valencia-A, S. 2004. Diversidad del género Quercus (Fagaceae) en México. Boletín de la Sociedad Botánica de México, 75: 33-53.

Recibido: 26 de abril 2018

Aceptado: 31 de mayo 2018 


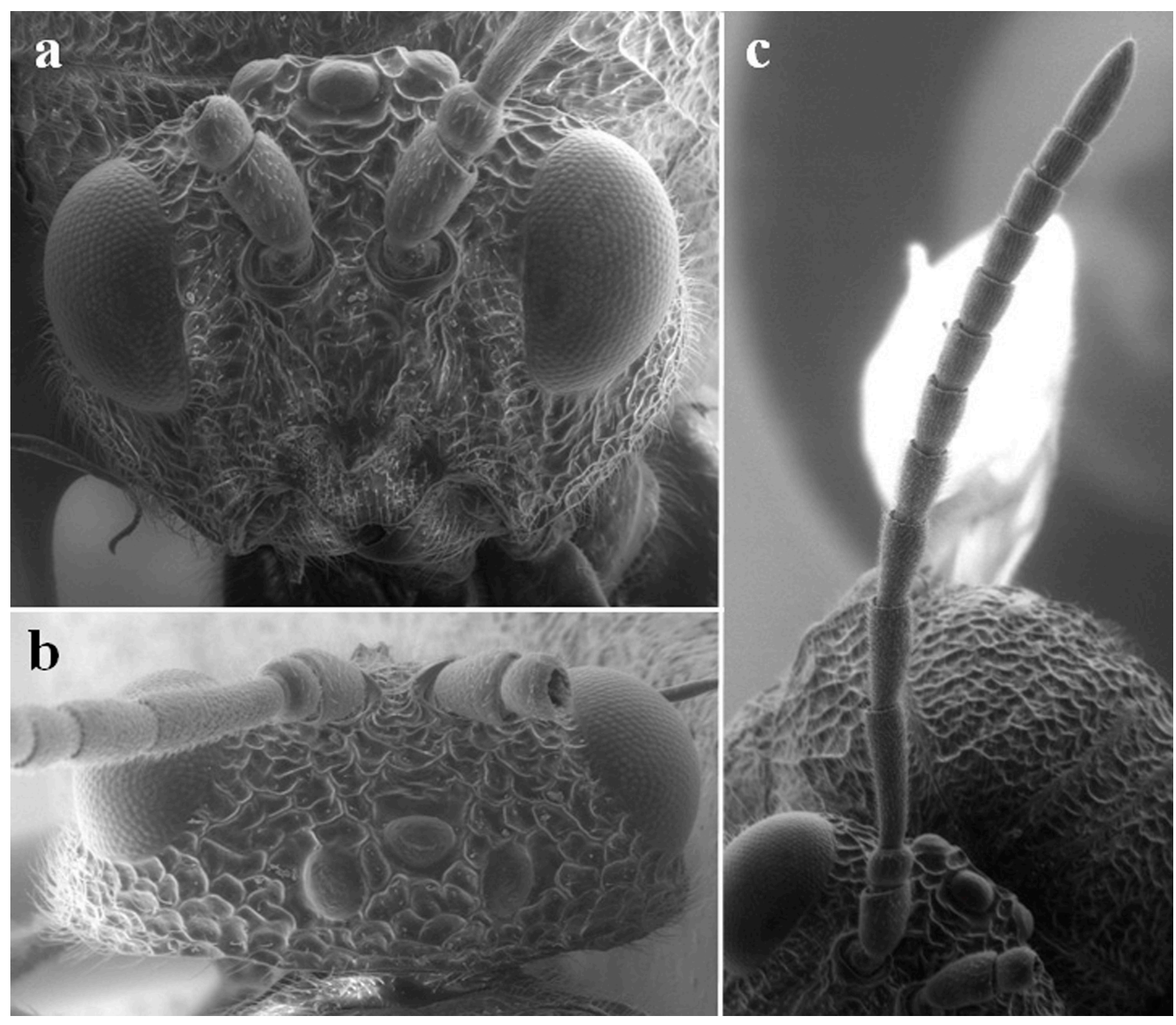

Figura 1. Amphibolips cibriani n. sp.: (a) cabeza en visión frontal, (b) cabeza en visión dorsal, (c) antena. 


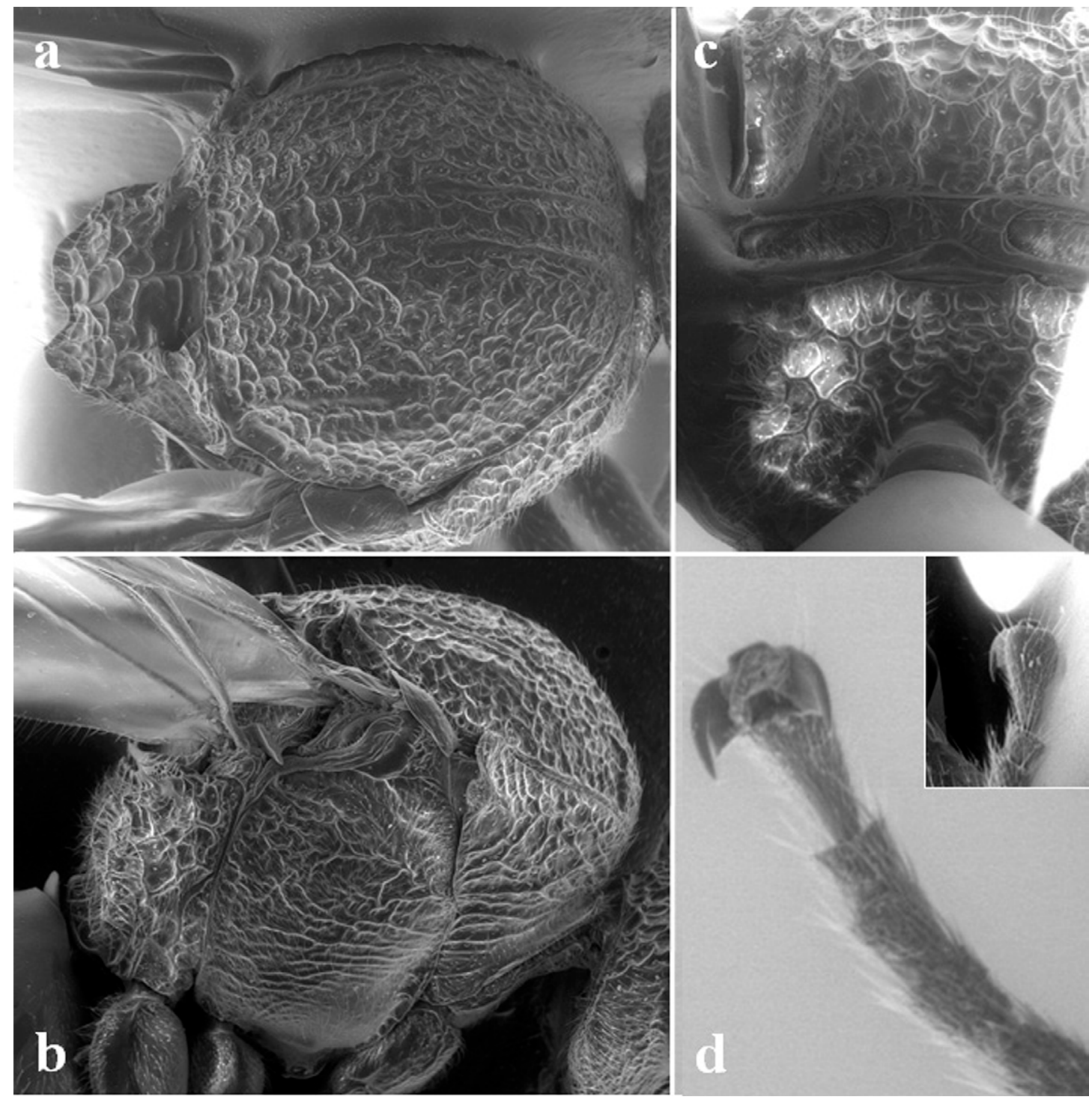

Figura 2. Amphibolips cibriani n. sp.: (a) mesosoma en visión dorsal, (b) mesosoma en visión lateral, (c) propodeo, (d) uñas tarsales. 


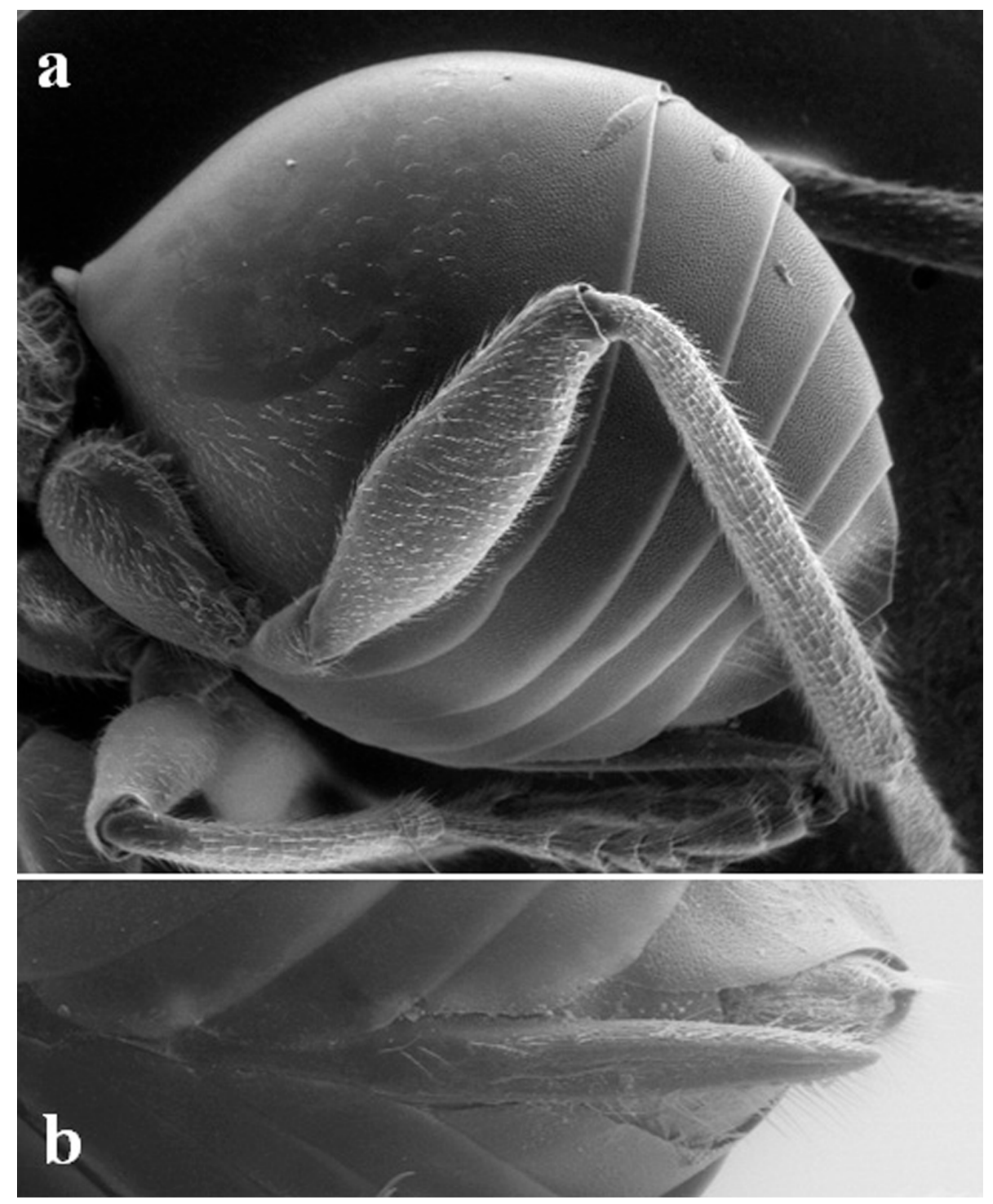

Figura 3. Amphibolips cibriani n. sp.: (a) metasoma en visión lateral, (b) espina ventral. 


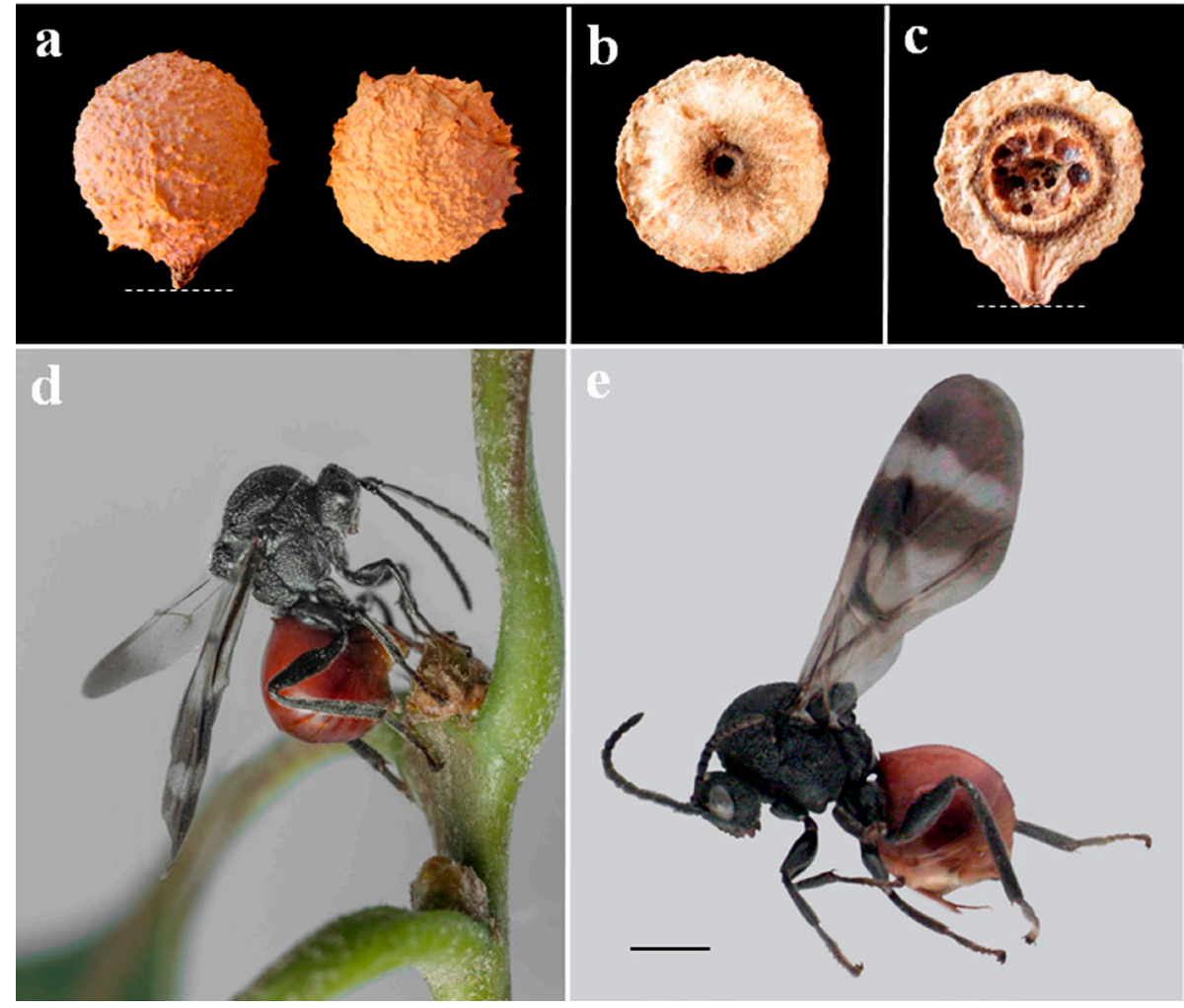

Figura 4. Amphibolips cibriani n. sp.: (a) aspecto general de las agallas, (b) corte transversal de una agalla, (c) ídem atacada por Synergus striatifrons, (d) adulto ovopositando en una yema de Q. crassipes, (e) habitus en visión lateral (escala $=1 \mathrm{~mm}$.). 